\title{
Current-temperature model of ACCC conductors based on GA identification method*
}

\author{
Xuan Tong and Xu-Dong Zhang \\ School of Energy and Power Engineering, \\ Nanjing University of Science and Technology \\ Nanjing 210093, China \\ E-mail: sunshine01014@ sina.com \\ www.njust.edu.cn
}

\begin{abstract}
As a new energy-saving conductor, ACCC conductor, which has the advantages of low weight, high conductivity, can double the ampacity of conventional conductors. However due to the differences between ACCC conductor and conventional conductors, present current-temperature model cannot be directly applied into the ampacity calculating of ACCC conductor. Consequently, the transmission capacity of ACCC conductor is limited. To address this problem, a current-temperature model for ACCC conductor based on thermal equilibrium principle and thermoelectricity analogy theory is proposed in this paper. Then, a GA identification method is employed to identify the model parameters. In order to validate the proposed model, an experiment platform is set up and the experiment results are presented and discussed.
\end{abstract}

Keywords: Energy-saving; ACCC Conductors; Current-Temperature Model; GA.

\section{Introduction}

Saving the energy of transmission lines is restricted by the ampacity of overhead conductors. Kinds of conductors have been put forward to increase the capacity of transporting electricity. Among these conductors, ACCC(Aluminum Conductor Composite Core) conductor is becoming a mature option to uprate line capacity for its advantages of low weight, low sag, high conductivity and high ampacity[1-2]. But owing to the fact that the ampacity of ACCC conductor is determined by static thermal rating so far has resulted in the limit on conductor's advantage in power transmission, it is essential to establish the current-temperature model for ACCC conductor to enhance its superiority of ampacity and energy-saving.

Current-temperature model refers to the mathematical relationship between temperature and ampacity, which is the foundation of line safety monitoring and

\footnotetext{
" This work is supported by "the Fundamental Research Funds for the Central Universities" No.30916011334.
} 
dynamic capacity-increasing[3-4]. Common current-temperature models can be mainly divided into standard model and thermal circuit model. The standard model includes IEC model and IEEE model[5-6], by which the ampacity of conductors may be estimated on condition of different weather as well as carrying current. While the thermal circuit model introduces thermoelectricity analogy theory into IEEE model to simplify the complicated heat transfer computation of conductors[7-9]. By this way, conductors' thermal dynamic process can be deduced through less parameters, elevating model's effectivity when applied into dynamic capacity-increase.

However, the current-temperature model aforementioned cannot be applied into ampacity evaluation of ACCC conductor for the unknown parameters caused by differences between ACCC conductor and conventional conductors on material, construction and running temperature. Meanwhile since there are few researches made on those parameters, the establishment of currenttemperature model for ACCC conductor has been delayed. In order to explore the potentiality of ACCC conductor ampacity, the current-temperature model of ACCC conductor based on thermal equilibrium theory and thermoelectricity analogy theory is established in this paper. And then GA identification method is proposed to compute the unknown parameters of model.

\section{Current-Temperature Model for ACCC Conductor}

According to the thermal equilibrium theory, the heat transfer between conductors and environment when operating can be described as

$$
C \frac{d T_{c}}{d t}=I^{2} R\left(T_{c}\right)+q_{s}-q_{c}-q_{r}
$$

Where, $t$ is time, $T_{c}$ is the conductor temperature, $C$ is the heat capacity of conductor, $I$ is loading current, $R\left(T_{c}\right)$ is the AC resistance of conductor at temperature $T_{c}, q_{s}$ is solar heat gain rate, $q_{c}$ is convection heat loss rate, $q_{r}$ is radiated heat loss rate.

Combined with thermoelectricity analogy theory, the thermal circuit model of ACCC conductor can be written as, with its circuit model shown in Figure 1:

$$
C \frac{d T_{c}}{d t}=I^{2} R\left(T_{c}\right)+q_{s}-\frac{\mathrm{T}_{c}-T_{e}}{R_{e}}-q_{r}
$$

Where, ${ }^{R_{e}}$ refers to thermal resistance. The parameters can be obtained by:

$$
\begin{gathered}
q_{s}=\gamma D S_{i} \\
q_{r}=\pi s D k_{e}\left(T_{c}^{4}-T_{e}^{4}\right)
\end{gathered}
$$




$$
R\left(T_{c}\right)=R_{r e f}\left(1+\alpha\left(T_{c}-T_{r e f}\right)\right)
$$

Where, $\alpha$ is resistance temperature coefficient, $R_{\text {ref }}$ is AC resistance at $T_{\text {ref }}$ that is always set at $20^{\circ} \mathrm{C}, D$ is conductor diameter, $S_{i}$ is solar intensity, $s$ is Stefan-Boltzmann constant, $\gamma=k_{e}=0.5$ are solar absorptivity and emissivity.

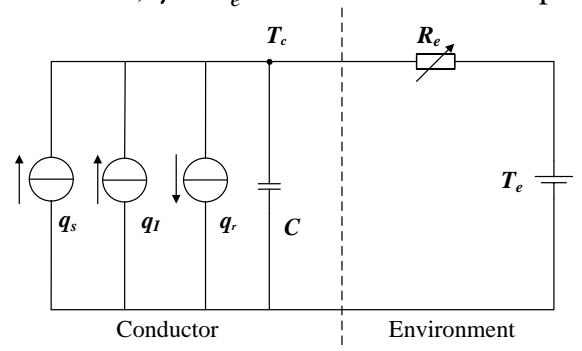

Fig. 1. Thermal circuit model of ACCC conductor.

\section{GA Identification Method for the Thermal Circuit Model}

As for ACCC conductor, the heat capacity $C$ cannot be obtained by theoretical method for the uncertain heat capacity of composite core. Also the AC resistance increasing introduced by eddy current and hysteresis can be neglected considering the insulation characteristic of core material. Therefore, the value rage of $R_{\text {ref }}$ and $\alpha$ for ACCC conductor have to be recalculated.

And according to [4], $R_{e}$ is related to the temperature difference between conductor and air essentially, it can be defined as

$$
R_{e}=a\left(T_{c}-T_{e}\right)^{b}
$$

Where, $a$ is scale factor related to conductor diameter and air density, $b$ is exponential factor. $a$ and $b$ need to be calculated by identification method.

To solve the aforementioned parameters, the thermal circuit model is discretized on time after introducing Eq.(3)-(6)

$$
T_{c}(k+1)=f_{k+1}\left(T_{c}(0), I(0), I(1), \ldots, I(k), R_{e}(k), T_{e}(0), T_{e}(1), \ldots, T_{e}(k), \mathbf{x}, \Delta t\right)
$$

Where, $\Delta t$ is time step, $\mathbf{x}$ represents the parameters vector to be identified

$$
\mathbf{x}=\left[C, \alpha, R_{r e f}, a, b\right]^{T}
$$

In this case, the objective function is proposed

$$
\begin{aligned}
& J(\mathbf{x})=\min \sum_{k=0}\left\|\tilde{T}_{c}(k+1)-T_{c}(k+1)\right\|^{2} \\
& \text { S.T } \quad \mathbf{x} \geq 0
\end{aligned}
$$

Where $\tilde{T}_{c}(k+1)$ refers to the measured temperature at time $k+1$. 
With the consideration of fine global search capability and fast convergence speed, GA is utilized to estimate the parameters for thermal circuit model. The GA is a powerful numerical optimization technique, which is rooted in the mechanism of natural evolution and genetics on the basis of natural selections. With the specific coding format in GA, the variables to be identified are composed of a set of genes which called as individual. A certain amount of individuals consist of a population which represents a solution set of optimization problem. The GA manipulates individual and generates successive populations using three basic operators: reproduction, crossover and mutation. The stronger individual has a great probability to mate with other stronger individual for producing stronger individual in successive population. Finally, the best survives are chosen as an optimization solution.

\section{Experimental Verification and Result Analysis}

\subsection{Experimental platform}

Figure 2 shows the temperature-rise experiment platform designed to solve the unknown parameters and verify its accuracy.

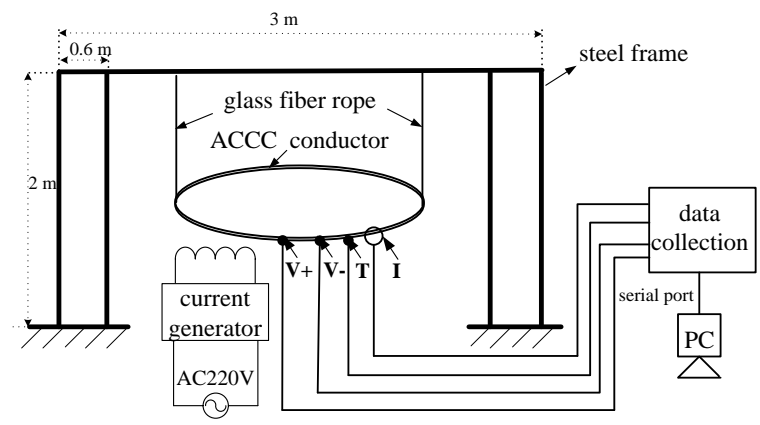

Fig. 2. The schematic diagram of temperature-rise experiment platform for ACCC conductor

The experiment platform is made up of steel frame, conductor ACCC240/35, current generator, temperature probe, voltage probe, Holzer current transformer, data collection apparatus and PC. And glass fiber rope is used to hang ACCC conductor for its thermo stability and low thermal conductivity. By positioning the experiment platform in the closed laboratory, the condition can be seen as natural convection.

\subsection{Result and analysis}

To acquire comprehensive temperature rise process, the current value of 400A to $1000 \mathrm{~A}$ are chosen as the carrying current. 
Introducing the temperature rise data into objective function, the value of unknown parameters, given by Table 1, can be obtained by GA.

Table 1. The identification results of model parameters

\begin{tabular}{ll}
\hline Model parameter & Identification result \\
\hline $\mathrm{C} /(\mathrm{J} \cdot \mathrm{K}-1 \cdot \mathrm{m}-1)$ & 789.13 \\
$\alpha$ & 0.0030 \\
$R_{\text {ref }}\left(\Omega \cdot \mathrm{km}^{-1}\right)$ & 0.1559 \\
$a$ & 0.7345 \\
$b$ & $-1.85 \times 10^{-8}$ \\
\hline
\end{tabular}

In order to verify the effectiveness of GA as an identification method, all the identified results are introduced into thermal circuit model to simulate thermal dynamic process under different carrying current, seen in Figure 3, the relative error is no more than $2.8 \%$.

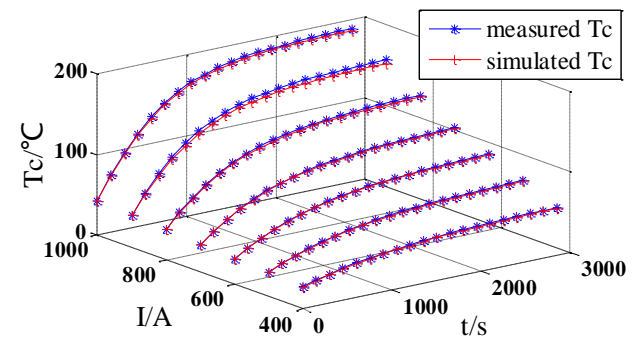

Fig. 3. The measured and simulated results of conductor temperature rise on different current

Then to further verify model accuracy, other current levels from 450A to $850 \mathrm{~A}$ are added. Figure 4 present the simulated result relative error, it illustrates that the model has high precision.

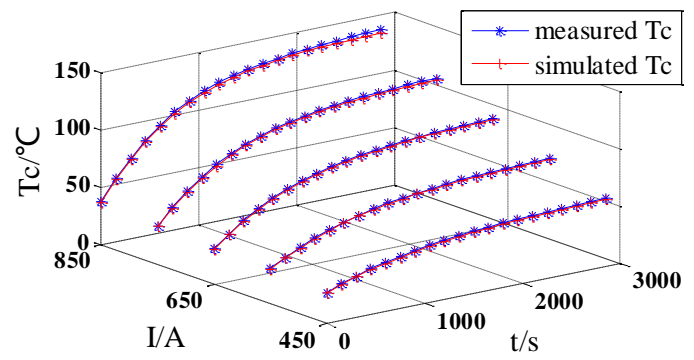

Fig. 4. The measured and simulated results of conductor temperature rise on added current.

\section{Conclusion}

The current-temperature model for ACCC conductor based on GA identification method is proposed in this paper. And the experiment results have shown that 
the GA identification method can solve the electrical and thermal parameters effectively. Meanwhile, in condition of natural convection, the model is high in precision, by which the temperature rise process can be simulated accurately to maximum the advantage on ampacity and energy-saving for ACCC conductor. Therefore, This will provide theoretical basis for further research on complicated meteorological conditions.

\section{References}

1. Burks B. Failure prediction analysis of an ACCC conductor subjected to thermal and mechanical stresses. Dielectrics and Electrical Insulation[J]. IEEE Transactions on Dielectrics and Electrical Insulation, 2010,17(2): 588-596.

2. I. Zamora, A. J. Mazón, P. Eguía, R. Criado, C. Alonso, J. Iglesias, and J. R. Sáenz. High-temperature conductors: A solution in the uprating of overhead transmission lines[M]. in Proc. Power Tech, 2001, vol. 4.

3. Cecchi V, Knudson M, Miu K. System impacts of temperature-dependent transmission line models[J]. IEEE Transactions on Power Delivery, 2014, 30(28):2300-2308.

4. IEC 61597-1995, Overhead electrical conductors -Calculation methods for stranded bare conductors[S]. International Electro technical Commission, 1995.

5. IEEE Std 738-2006, IEEE Standard for Calculating the CurrentTemperature of Bare Overhead Conductors[S]. New York, Institute of Electrical and Electronics Engineers, 2006.

6. LIU Gang, RUAN Banyi, ZHANG Ming. A Transient Model for Overhead Transmission Line Dynamic Rating Based on Thermal Circuit Method[J]. Automation of Electric Power Systems, 2012, 36(16): 58-62,123.

7. LIU Gang, RUAN Banyil, LIN Jie, YANG Min, et al. Steady-state Model of Thermal Circuit Method for Dynamie Overhead Lines Rating[J]. High Voltage Engineering, 2013, 39(5): 1107-1113.

8. Boguslaw Wiecek, Gilbert De Mey, Vasilis Chatziathanasiou, Antonis Papagiannakis, et, al. Harmonic analysis of dynamic thermal problems in high voltage overhead transmission lines and buried cables[J]. Electrical Power and Energy Systems, 2014, 58: 199-205.

9. Alawar A, Bosze E J, Nutt S R. A composite core conductor for low sag at high temperatures[J]. IEEE Transactions on Power Delivery, 2005, 20(3):2193-2199. 